\title{
REMARKS ON WEAK TYPE INEQUALITIES FOR OPERATORS COMMUTING WITH TRANSLATIONS
}

\author{
BY R. R. COIFMAN
}

Communicated by A. P. Calderon, November 30, 1967

The purpose of this note is to show that by very simple arguments one can obtain an analogue of E. M. Stein's theorem [1] for noncompact $\sigma$-compact groups. Together with Theorem 1 of Stein we get the following

THeOReM I. Let $G$ be a locally compact $\sigma$-compact group. $T_{m}$ a sequence of bounded linear operators of $L^{p}(G)$ into itself $1 \leqq p \leqq 2$ such that

(a) $T_{m}\left(f_{g}\right)(x)=T_{m}(f)(g x)$ where $f(g x)=f_{\theta}(x)$.

(b) The support of $T_{m}(f)$ is contained in a compact set whenever $f$ has compact support.

Define $M(f)(x)=\sup _{m}\left|T_{m}(f)(x)\right|$. Then the following conditions are equivalent:

$1^{\circ}$. $\forall f \in L^{p}(G) M(f)(x)<\infty$ a.e. and $|\{x: M(f)(x)>\lambda\}|<\infty$ for some $\lambda$ (depending on $f$ ).

$2^{\circ}$. $|\{x: M(f)(x)>\lambda\}| \leqq C\left(\|f\|_{p} / \lambda\right)^{p}$ for $\lambda \geqq\|f\|_{p}$ where $C$ is independent of $f$.

Here $|E|$ denotes the left Haar measure of the set $E$.

Moreover, if $G$ is not compact, the restriction $p \leqq 2$ is not necessary.

It is evident that Theorem I reduces to Theorem 1 in [1] when $G$ is compact. We are going to consider the case $G$ noncompact. As will be seen from an example the restriction $\|f\|_{p} \leqq \lambda$ is necessary. However, if we replace condition (a) by condition $3^{\circ}$ of Theorem II below we get a global weak type estimate.

Definition. $A$ is an affine map on $G$ if it can be represented as a composition of left and right translations with continuous automorphisms of $G$.

From the uniqueness of left Haar measure

$$
\Delta_{A} \int_{G} f_{A}(x) d x=\int_{G} f(x) d x \text { where } f_{A}=f(A x)
$$

for some constant $\Delta_{A}>0$.

Theorem II. Let $M$ be a sublinear operator ${ }^{1}$ defined on $L^{p}(G)$,

${ }^{1} M$ is sublinear if and only if $|M(f+g)(x)| \leqq|M(f)(x)|+|M(g)(x)|$ and $|M(\lambda f)(x)|=|\lambda||M(f)(x)|$. 
$1 \leqq p \leqq \infty$, into measurable functions such that

$1^{\circ}$. $\forall f \in L^{p}, M(f)(x)<\infty$ a. e. and $|\{x: M(f)(x)>\lambda\}|<\infty$ for some $\lambda$.

$2^{\circ}$. If $f_{k} \rightarrow f$ in norm in $L^{p}$ then there exists a subsequence $f_{i_{k}}$ such that

$$
M(f)(x) \leqq \liminf _{k \rightarrow \infty} M\left(f_{i_{k}}\right)(x) \quad \text { a.e. }
$$

$3^{\circ}$. There exists an affine map $A$ with $\Delta_{A} \neq 1$ such that

$$
M\left(f_{A}\right)(x)=\Delta_{A}^{\alpha} M(f)(A x) \quad \text { for some } \alpha \in R
$$

then

$$
|\{x: M(f)(x)>\lambda\}| \leqq C\left(\|f\|_{p} / \lambda\right)^{q},
$$

where $1 / q=1 / p+\alpha$.

REMARK. The condition $\Delta_{A} \neq 1$ implies already that $G$ cannot be compact. We will prove later that condition $2^{\circ}$ is verified for $M$ in Theorem I.

The proof is based on the following lemma of Edwards and Hewitt [2].

Lemma (EDwards-HewitT [2, Theorem $(1,5)]$ ). Let $M$ satisfy conditions $1^{\circ}$ and $2^{\circ}$ of Theorem II. Then

$$
\begin{gathered}
\forall f \in L^{p}, \quad \forall \lambda>0, \quad \text { there exists } C(\lambda) \text { such that } \\
\left|\left\{x: M(f)(x)>C(\lambda)\|f\|_{p}\right\}\right| \leqq \lambda .
\end{gathered}
$$

Proof. Condition $1^{\circ}$ is clearly equivalent to the following:

$1^{\circ \prime} . \forall f \in L^{p},|\{x: M(f)(x)>\lambda\}| \rightarrow 0, \lambda \rightarrow \infty$.

Let $E_{n, \lambda}=\left\{f \in L^{p}:|\{x: M(f)(x)>n\}| \leqq \lambda\right\}$, then $1^{\circ \prime}$ implies $\bigcup_{n=0}^{\infty} E_{n, \lambda}=L^{p}$. Moreover, $E_{n, \lambda}$ are closed (this follows immediately form $2^{\circ}$ ). Applying Baire's Category Theorem there exists $E_{n_{\lambda}, \lambda}$ containing a ball $S_{\lambda}$ of radius $r_{\lambda}$ centered at $f$. Clearly every element of the ball in $L^{p}$ of radius $r_{\lambda}$ centered at 0 is a difference of 2 functions in $E_{n_{\lambda}, \lambda}$ so that (1.1) holds for some constant $C_{\lambda}$.

Proof of Theorem II. Let

$$
C(\lambda)=\inf \left\{C_{\lambda} \geqq 0: \forall f \in L^{p},\left|\left\{x: M(f)(x)>C_{\lambda}\|f\|_{p}\right\}\right| \leqq \lambda\right\},
$$

then

$$
\left|\left\{x: M\left(f_{A}\right)(x)>C(\lambda)\left\|f_{A}\right\|_{p}\right\}\right| \leqq \lambda
$$

but $\left\|f_{\Lambda}\right\|_{p}=\Delta_{\Lambda}^{-1 / p}\|f\|_{p}$ and condition $3^{\circ}$ imply 


$$
\begin{aligned}
\Delta^{-1} \mid\{x: M(f)(x) & \left.>C(\lambda) \Delta^{-(\alpha+1 / p)}\|f\|_{p}\right\} \mid \\
& =\left|\left\{x: M(f)(A x)>C(\lambda) \Delta^{-(\alpha+1 / p)}\|f\|_{p}\right\}\right| \\
& =\left|\left\{x: M\left(f_{A}\right)(x)>C(\lambda)\left\|f_{A}\right\|_{p}\right\}\right| \leqq \lambda
\end{aligned}
$$

thus

$$
C(\lambda \Delta) \leqq C(\lambda) \Delta^{-(\alpha+1 / p)} .
$$

Clearly $3^{\circ}$ holds for $A$ replaced by $A^{k}$ ( $k$ integer) and $\Delta$ by $\Delta^{k}$ so that

$$
C\left(\lambda \Delta^{k}\right) \leqq C(\lambda)\left(\Delta^{k}\right)^{-(\alpha+1 / p)} .
$$

Since $C(\lambda)$ is decreasing we get $C(\lambda) \leqq C \lambda^{-(\alpha+1 / p)}$. Q.E.D.

Proof of Theorem I. Let us prove first that condition $2^{\circ}$ of Theorem II is verified. Let $f_{k} \rightarrow f$ in $L^{p}$; since $T_{n}$ is continuous in measure, we can extract a subsequence such that $T_{n}\left(f_{k_{i}}\right)(x) \rightarrow T_{n}(f)(x)$ a.e.; by a diagonalization process we can choose the subsequence independent of $n$. Assume now that

$M(f)(x)>\lambda$; there exists $n(x)$ such that $T_{n(x)}(f)(x)>\lambda$, so there exists $k(x)$ such that

$$
k>k(x) \Rightarrow T_{n(x)}\left(f_{k}\right)(x)>\lambda,
$$

which implies

$$
M\left(f_{k}\right)(x)>\lambda,
$$

hence $\lim \inf _{k \rightarrow \infty} M(f)(x) \geqq \lambda$.

Using the lemma we get

$$
\left|\left\{x: M(f)(x)>C(\lambda)\|f\|_{p}\right\}\right| \leqq \lambda .
$$

Our purpose is to compute $C(\lambda)$. Define

$$
M_{N}(f)(x)=\sup _{n \leqq N}\left|T_{n}(f)(x)\right|
$$

then

$$
\left|\left\{x: M_{N}(f)(x)>C(\lambda)\left\|_{f}\right\|_{p}\right\}\right| \leqq\left|\left\{x: M(f)(x)>C(\lambda)\|f\|_{p}\right\}\right| \leqq \lambda
$$

and

$$
\begin{aligned}
\left|\left\{x: M_{N}(f)(x)>C(\lambda)\|f\|_{p}\right\}\right| \rightarrow\left|\left\{x: M(f)(x)>C(\lambda)\|f\|_{p}\right\}\right|, & \\
N & \rightarrow \infty .
\end{aligned}
$$

We now need the following simple lemma.

Lemma. Let $G$ be a locally compact noncompact group. Let $K$ be a compact subset, then there exists $h \in G$ such that $h K \cap K=\varnothing$. 
Proof. Let $L$ be the union of all $h K$ for which $h K \cap K \neq \varnothing$. Clearly $L \subseteq K K^{-1} K$, which is compact. If the lemma is false then $L=G$ compact, contrary to our assumption.

Let $f \in L^{p}$ have compact support. By the lemma, there exists $h_{N} \in G$ such that supp $f \cap \operatorname{supp} f_{h_{N}}=\varnothing$ and supp $T_{m} f \cap$ supp $T_{m}(f)_{h_{N}}$ $=\varnothing$ for all $m \leqq N$. Thus

$$
\begin{aligned}
M_{N}\left(f+f_{h_{N}}\right) & =M_{N}(f)+M_{N}(f)_{h_{N}}, \text { and supp } M_{N}(f) \cap \operatorname{supp} M_{N}\left(f_{h_{N}}\right)=\varnothing . \\
\lambda & \geqq\left|\left\{x: M_{N}\left(f+f_{h_{N}}\right)(x)>\left\|f+f_{h_{N}}\right\|_{p} C(\lambda)\right\}\right| \\
& =\left|\left\{x: M_{N}(f)(x)+M_{N}(f)\left(h_{N} x\right)>2^{1 / p}\|f\|_{p} C(\lambda)\right\}\right| \\
& =2\left|\left\{x: M_{N}(f)(x)>2^{1 / p} C(\lambda)\|f\|_{p}\right\}\right| .
\end{aligned}
$$

Repeating the argument for $k$ translates, we get

$$
\left|\left\{x: M(f)(x)>k^{1 / p} C(\lambda)\|f\|_{p}\right\}\right| \leqq \lambda / k, \quad k>0 \text { integer }
$$

for all $f$ with compact support. Using $2^{\circ}$ of Theorem II, we extend the inequality for all $f \in L^{p}$ and the theorem follows.

REMark. We proved Theorem I under the assumption $T_{m}$ are continuous in measure.

EXAMPLE. Let

$$
M(f)(x)=\int_{R^{n}} \frac{f(x-y)}{|y|^{\alpha}} d y, \quad 0<\alpha<n, f \in L^{p} .
$$

Then

$$
M(f)(x)=\int_{|y| \leqslant 1} \frac{f(x-y)}{|y|^{\alpha}} d y+\int_{|y|>1} \frac{f(x-y)}{|y|^{\alpha}} d y .
$$

The first integral is a convolution with an integrable function so it belongs to $L^{p}$. The second is a convolution with an $L^{p_{1}}$ function for $p_{1}>n / \alpha$ so it belongs to $L^{q_{1}}, 1 / q_{1}=1 / p+1 / p_{1}-1$. Thus condition $1^{\circ}$ of Theorem II is verified. Moreover, it is clear that

$$
M\left(f_{\lambda}\right)(x)=\int_{R^{n}} \frac{f(\lambda x)}{|x-y|^{\alpha}} d y=\lambda^{-n+\alpha} M(f)(\lambda x)
$$

so that condition $3^{\circ}$ of Theorem II is verified (the affine map is a dilation). By Theorem I, $M$ maps $L^{p}$ into weak $L^{q}$ continuously for $1 / q=1 / p+\alpha / n-1$.

We can easily improve the result to get a theorem of Stein and Weiss [3]. Consider

$$
M(f)(x)=\frac{1}{|x|^{\gamma}} \int_{R^{n}} \frac{f(y)}{|x-y|^{\beta}|y|^{\alpha}} d y
$$


where $f \in L^{p}, 0<\beta<n, \alpha<n(1-1 / p), \alpha+\gamma \geqq 0,1 / p-1+(\alpha+\beta+\gamma) / n$ $>0$. Then

$$
M\left(f_{\lambda}\right)(x)=\frac{1}{|x|^{\gamma}} \int_{R^{n}} \frac{f(\lambda y)}{|x-y|^{\beta}|y|^{\alpha}} d y=\lambda^{-n+\alpha+\beta+\gamma} M(f)(\lambda x) .
$$

So that $M$ will map $L^{p}$ into weak $L^{q}, 1 / q=1 / p+(\alpha+\beta+\gamma) / n-1$ if we can prove $1^{\circ}$ Theorem II. But

$$
\begin{aligned}
M(f)(x)= & \frac{1}{|x|^{\gamma}} \int_{|y|<|x| / 2} \frac{f(y)}{|x-y|^{\beta}|y|^{\alpha}} d y \\
& +\frac{1}{|x|^{\alpha}} \int_{|x| / 2 \leqq y} \frac{f(y)}{|x-y|^{\beta}|y|^{\alpha}} d y .
\end{aligned}
$$

Now if $|y|<\frac{1}{2}|x|$ then $|x-y|>\frac{1}{2}|x|$ so that

$$
M(f)(x) \leqq \frac{C}{|x|^{\beta+\gamma}} \int_{|y|<|x| / 2} \frac{|f(y)|}{|y|^{\alpha}} d y+\frac{1}{|x|^{\alpha+\gamma}} \int_{R^{n}} \frac{|f(y)|}{|x-y|^{\beta}} d y .
$$

Applying Holder's inequality to the first integral for $\alpha<n(1-1 / p)$, we get

$$
M(f)(x) \leqq \frac{C\|f\|_{p}}{|x|^{\alpha+\beta+\gamma-n(1-1 / p)}}+\frac{1}{|x|^{\alpha+\gamma}} \int \frac{|f(y)|}{|x-y|^{\beta}} d y .
$$

It is clear, using the previous example and conditions on $\alpha, \beta, \gamma$, that $1^{\circ}$ is verified.

Remark. To see that the restriction $\|f\|_{p} \leqq \lambda$ in Theorem I is essential let us consider

$$
T_{n}(f)(x)=\int_{1 / n<|y|<n} \frac{f(x-y)}{|y|^{a}} d y .
$$

Then $T_{n}$ maps $L^{p}$ continuously into itself, $M(f)$, however, will map $L^{p}$ into weak $L^{q}, 1 / q=1 / p+\alpha / n-1$, i.e. $q>p$, and by the proof of Theorem II, will be identically 0 if the inequality of Theorem I were to hold without the restriction $\|f\|_{p} \leqq \lambda$.

\section{REFERENCES}

1. E. M. Stein, On limits of sequences of operators, Ann. of Math. 74 (1961), 140-170.

2. R. E. Edwards and E. Hewitt, Pointwise limits for sequences of convolution operators, Acta Math. 113 (1965), 181-218.

3. E. M. Stein and Guido Weiss, Fractional integrals on n-dimensional Euclidean space, J. Math. Mech. 7 (1958), 503-514.

University of Chicago 